Tropical Journal of Pharmaceutical Research, June 2006; 5 (1): 545-550

(C) Pharmacotherapy Group,

Faculty of Pharmacy, University of Benin

Benin City, Nigeria.

All rights reserved.

Research Article

Available online at http://www.tjpr.org

\title{
Identification of Counterfeit Drugs by Community Pharmacists in Lagos State
}

\section{*a Odili, Valentine U., ' Osemwenkha Sylvia, ${ }^{\mathrm{c} E k e}$ Esther U. and dOkeri Henry A.}

aDept, of Clinical Pharmacy \& Pharmacy Practice, Faculty of Pharmacy, University of Benin. Benin City,

${ }^{b}$ Institute of Public Administration \& Extension Services, University of Benin, Benin City.

${ }^{\mathrm{c}, \mathrm{d}}$ Dept. of Pharmaceutical Chemistry, Faculty of Pharmacy, University of Benin, Benin City.

\begin{abstract}
Purpose: The problem of fake and counterfeit drugs is real and constitutes a major threat to the health and safety of the Nigerian population. A descriptive study was carried out to assess the methods of identification of counterfeit drugs by community pharmacists in Lagos State.

Methods: The research instrument was a 23-item questionnaire, which was administered to consenting community pharmacists in 17 out of the 20 Local Government Areas in Lagos State.

A convenient sample of practicing community pharmacists both in retail and wholesale distribution of pharmaceutical products were recruited into the study using a list of registered pharmacists from the Association of Community Pharmacists in Lagos State. Effort was made to ensure adequate representation of Pharmacies in every local government area in Lagos State.

Results All the respondents agreed that there is a fake and counterfeit drug problem in Nigeria, and (74\%) considered this a major problem. The respondents commonly used visual security techniques before drug purchasing. These were: Seals/embossments (83\%), character of print (77\%), and Holograms (68\%).The respondents' most likely action after a counterfeit drug encounter was to return the drug back to the supplier.

Conclusion: The study showed that pharmacists sampled were aware of the prevalence of fake and counterfeit drugs and quite a number of them have had encounters with them. There is an indication that the respondents try to assure themselves of the quality of the drugs they purchase by use of several methods. However, no rigorous effort was taken to confirm as well as report suspected fake and counterfeit drugs to regulatory authorities.
\end{abstract}

KEYWORDS: Community pharmacists, drug identification, fake and counterfeit drugs.

*Corresponding Author: E-mail: vuodili@yahoo.com +2348023432237 


\section{INTRODUCTION}

The problem of fake and counterfeit drugs exists in both developed and developing countries. From January 1999 to October 2000, the World Health Organisation (WHO) received 46 confidential reports of counterfeit drugs from 20 countries. Of these, $60 \%$ came from developing countries. ${ }^{(1)}$ Counterfeiting of drugs has become highly profitable and counterfeiters have seriously improved on their cloning of genuine drugs because of recent technological advances A counterfeit drug is one, which is deliberately, fraudulently mislabelled with respect to identity and or source. ${ }^{(2)}$ Counterfeiting could be in the form of insufficient amounts of ingredients, incorrect ingredients, lack of active ingredients or fake packaging.

In Nigeria, the National Agency for Food and Drug Administration and Control (NAFDAC) has enlarged the scope of counterfeit drugs to include drugs without the full name and address of manufacturers, drugs labelled "For Export Only", expired and relabelled drugs with the intention of extending their shelf lives, drugs containing banned substances and drugs not registered by NAFDAC. ${ }^{(3)}$

The World Health Organisation (WHO) has estimated that $10 \%$ of global pharmaceutical commerce is in counterfeit drugs and is present in all countries. ${ }^{(4,5,6)}$ Informed estimates put the size of the annual global market in fake drugs in the region of USD30 billion, which represents about $7 \%$ of pharmaceutical industry revenue. $(7,8) \quad$ A survey of pharmacies in the Philippines showed that $8 \%$ of drugs bought were fake, another survey done in five countries in mainland South East Asia reported that $40 \%$ of tablets sold as the new antimalarial Artesunate were fake. ${ }^{(8,9)}$

Pharmacists are the custodians of drugs and have the responsibility for sourcing, manufacturing, distributing, dispensing and monitoring the outcome of drug therapy on users. Sadly, this is strictly not so in the Nigerian context where drug distribution is chaotic and has become an all-comers affair. Pharmacists find themselves in the same situation as the members of lay public who have no clue whether the drug they have purchased is genuine or fake.

To our knowledge, there has been no study carried out to investigate how pharmacists, particularly in the community setting identify or differentiate between genuine and fake I counterfeit drug products.

The objectives of this study were to: determine the frequency of fake and counterfeit drug experiences; identify the most common methods of identifying such drug products; assess the use of visual clues in identification of fake and counterfeit drugs and to determine likely action following the detection of counterfeit drugs by community pharmacists in Lagos State, Nigeria.

\section{METHODS \\ Setting}

Data was collected for this study from 17 out of the 20 statutory local government areas (LGAs) in Lagos State. The sample was chosen to represent the spread of community pharmacists registered in Lagos State. Lagos Island, IpejuLekki and Epe were excluded due to nonavailability of pharmacists in those areas.

Lagos State was selected because it is the major distribution centre for imported pharmaceutical products and has the largest concentration of pharmaceutical manufacturers, as well as community pharmacists in Nigeria.

\section{SAMPLE}

A convenient sample of 69 practicing community pharmacists, both in retail and wholesale distribution of pharmaceutical products were recruited for the study, using a list of registered pharmacists from the Association of Community Pharmacists in Lagos State as the sampling frame. From this list, a simple random selection procedure by use of a ballot was done per LGA, in order to ensure adequate representation of pharmacies in every LGA in the state.

\section{Questionnaire design and administration}

The research instrument had two sections. Section A was designed to elicit demographic information of the respondents such as age, location, type of pharmacy and number of years in practice. Section B was made up of 17 questions, some of the questions required a 
simple Yes or No response e.g. "I have had an experience stocking fake and counterfeit drugs in my pharmacy", "I usually procure drugs for my pharmacy", "I check on the source of drugs before procurement", "I have reported a case of fake and counterfeit drugs to the regulatory authorities" "Nigeria has a fake and counterfeit drug problem". The responses to a few questions were anchored on an ordinal scale e.g. for the question "I would classify my experiences of fake and counterfeit drugs as being:"options were very often, often, seldom and none. The respondents were asked in another question to classify the fake and counterfeit drug problem in Nigeria as: major, moderate, minor or none.

The remaining questions focused on other objectives of the study such as; the methods employed by respondents to detect fake drugs, factors that determine where they source their drugs, their most frequent sources of drug procurement, visual and regulatory security marks that guided their drug purchases as well their likely responses after a fake drug encounter.

\section{DATA ANALYSIS}

Data was entered into EPI Info version 6 and descriptive statistics were computed for all quantitative variables.

\section{RESULTS \\ Demographics}

Overall, 100 copies of the questionnaire were distributed to the community pharmacists and 69 completed usable questionnaires were received, giving a response rate of $69 \%$.

About two - thirds of the respondents $65.2 \%$ $(45 / 69)$ were males, majority of the respondents $73.3 \%$ were aged between 31 - 50 years, with a mean age of 41.7 years. Most of the respondents $57,(82.6 \%)$ were in retail practice, while only $12(17.4 \%)$ were in wholesale practice. Only $21.7 \%$ of them had additional qualifications. The mean period of practice experience was 17.3 years.

All the respondents agreed that Nigeria has a fake and counterfeit drug problem; 51 (74\%) of the respondents went further to describe the problem as major. The remaining 18 (26\%) thought it was moderate. Some 59 (86\%) of the respondents procured their drug products personally, while 10 (14.5\%) were not personally involved in drug procurement. 52 (75\%) of respondents sourced their drugs from middlemen and drug wholesalers. Other sources were the "open markets" and pharmaceutical company representatives, (Table 1). Fifty-six $(81 \%)$ of the respondents agreed that they checked on the source of their drugs before procurement. The factors that determined where respondents made their purchases included the reputation of the distributor (62\%) and a personal good feeling that the product is genuine (41\%). Others are as shown in Table 1.

About 42 respondents (61\%) had experienced stocking some counterfeit products in their premises. Scrutiny of purchased drug products was indicated as the commonest means of screening for fake and counterfeit drugs by $51 \%$ of respondents. Other methods were customers' complaints (10.1\%) and alerts from pharmaceutical companies (7.3\%). No respondent reported ever receiving any help from regulatory officials in detecting fake and counterfeit drugs.

The visual security guides used by respondents included seals and tablet embossments, which had the highest guide rate (83\%), this was followed by character of print (77\%).

Other methods such as holograms, colour of package and colour of product were also used but to a relatively less extent.

As regards detecting fake drugs by use of regulatory markings on drug packaging, majority (83\%) of the respondents checked for NAFDAC registration numbers while manufacturers' name/address and expiry/date of manufacture of drug were checked by $71 \%$ and $69 \%$ of respondents respectively. The check for batch number was the least and was done by only about $39 \%$ of respondents

Fifty-six (81\%) of the respondents had never reported any case of fake and counterfeit drug encounter to the regulatory authorities. The most likely action of the respondents after an 
Table I: Responses to issues relating to drug sourcing and counterfeit drug detection.

\begin{tabular}{lcc}
\hline & \multicolumn{2}{c}{ Frequency $^{(\%)^{\mathbf{a}}}$} \\
\hline Methods of detecting fake drugs by respondents & 7 & 10.1 \\
Customers & 35 & 50.7 \\
Close scrutiny & 0 & 0 \\
Regulatory officials & 5 & 7.3 \\
News/company alerts & 28 & 40.6 \\
No response & & \\
Determinants of where to source drugs & 28 & 40.6 \\
Good feeling & 43 & 62.3 \\
Distributor & 32 & 46.4 \\
Medical Representative & 14 & 20.3 \\
References from other pharmacists & & \\
Most frequent source of drug procurement & 11 & 15.9 \\
Open market & 39 & 56.5 \\
Medical representative & 52 & 75.4 \\
Middle men/ Wholesalers & & \\
Visual security checks which guide respondent's purchases & 47 & 68.1 \\
Holograms & 57 & 83.0 \\
Seals/Embossments & 53 & 77.0 \\
Character of print & 42 & 61.0 \\
Colour of package & 41 & 59.4 \\
Colour of drug product & 4 & 5.8 \\
No response & & \\
Regulatory marks that guide respondent's purchases & 60 & 87.0 \\
NAFDAC number & 27 & 39.1 \\
Batch number & 49 & 71.0 \\
Manufacturers' name address & 48 & 69.0 \\
Date of manufacture/Expiry & 2 & 2.9 \\
No response & & \\
\hline a multiple responses were allowed hence total above 100\%.9.
\end{tabular}

${ }^{a}$ multiple responses were allowed hence total above $100 \%$.

encounter included 'taking the product back to the supplier' (65.2\%), 'reporting to professional bodies' $(50.7 \%)$, 'taking the product back to its distributor' (11.6\%), and 'reporting to NAFDAC' (18.8\%). A few of the respondents (11.6\%) did not respond.

Results of the assessment of the impact of NAFDAC at curbing the proliferation of counterfeit drugs showed that $18.8 \%$ of the respondents thought they were very effective; but the majority (63.8\%) thought they were only moderately effective while $15.9 \%$ thought they were ineffective.

\section{DISCUSSION}

The problem of fake and counterfeit drugs is widespread with a high global prevalence. Reports from the pharmaceutical industry and governments clearly indicate that the methods and channels used by counterfeiters are now more sophisticated, thus making counterfeit medicines difficult to detect. ${ }^{(4,10,11,12)}$

This survey shows that majority of the respondents have experienced stocking some counterfeit drug products in their premises. The common means by which they screen for phoney products was by close visual scrutiny. This finding is very encouraging as it suggests that the respondents are aware of the high occurrence of fake drugs and are motivated to 
check for drug security measures in order to identify such products. However, it is pertinent to note that fake and counterfeit drug detection using only visual inspection is not foolproof and so is unreliable except when the suspected drug is from unsophisticated forgers.

All the respondents agreed that Nigeria has a fake and counterfeit drug problem and majority of them described this problem as being major. This is consistent with findings in several publications. $(7,8,10)$

Another interesting finding in this survey is the fact that majority of the respondents claimed they sourced their drug products personally from wholesalers. This is quite cheering considering the fact that only few of the respondents patronized the open drug markets, which has become a singular major source of pharmaceutical products in Nigeria. However, there is a worrying concern about where the wholesalers get their products. There is proof that major pharmaceutical distributors and even medical representatives dump their products in unlicensed open markets in order to meet sales targets and make quick turnover ${ }^{(4,10)}$

It is commendable that majority of the respondents are critical of the sources of drugs before procurement, but the factors that determine where they eventually make purchases is not so laudable. This survey revealed that the determinants as to choice of sources of purchase include the reputation of supplier and the purchasing pharmacist's "good feeling" that the products carried by the source are genuine. Some of these determinants as reported by the respondents are highly subjective and are based on the respondent's experience.

As regard regulatory marks, NAFDAC registration number, manufacturer's name/ address were the most commonly checked regulatory marks before drug purchase by respondents. The product batch number was the least checked regulatory mark on drug products. The reason for this was taught to be that a batch number in itself does not protect against purchasing fake and counterfeit drugs except of course when there is a ridiculous large quantity of the same batch number or when there has been an alert that drugs with a particular batch number are fake ${ }^{(13)}$

NAFDAC registration number on drug products accounted for the highest regulatory mark check rate by the respondents, this is not surprising because in Nigeria, product registration leads to the issuance of a NAFDAC number which is an administrative tool used in the limiting the number and types of regulated, imported or manufactured drugs in the country. It also aids in the introduction of new products as well as removal of unsuitable products from the market. The presence of NAFDAC number is part of the climax of all built in processes designed to ensure that drugs are safe, efficacious and of good quality. Therefore the reliance on NAFDAC number, as a security guide by respondents is praiseworthy. However, these numbers have been known to be faked and so it behoves the regulatory Agency to intensify and regularly inspect drug outlets in the country, in order to identify and publicize such products.

This study reveals that only few of the respondents have ever reported a case of fake and counterfeit drug to the authorities. This is disheartening considering the fact that majority of the respondents have had encounters with fake and counterfeit drug products in their practices. The preferred action of the respondents after an encounter was to return the fake product to the supplier rather than making a report to the regulatory authorities or destroying the product. This action prevents any possible arrest or prosecution of the culprit as well as encourages subsequent redistribution of the product. It is believed that the respondents prefer to return suspected spurious products to suppliers because, this way, they are not likely to lose the capital invested in such purchases, unlike when they choose the alternative of reporting or destroying the suspected products.

Furthermore, majority of respondents in this study assessed NAFDAC's activities at curbing the proliferation of fake and counterfeit drugs as only moderately effective this may also be a factor why majority of the respondents do not 
collaborate with NAFDAC after a fake and counterfeit drug encounter.

\section{LIMITATIONS}

The use of a convenient sample of pharmacists in Lagos state would limit the extent to which the study sample was representative of the opinions and practices of the general population of pharmacists at large. Also non-respondent data were not collected and therefore no characterizations can be made about them.

\section{CONCLUSION}

Pharmacists sampled are aware of the high prevalence of fake and counterfeit drugs and quite a number of them have had encounters with them. There is an indication that the respondents try to assure themselves of the quality of the drugs they purchase by use of several methods. However, no rigorous effort was taken to confirm as well as report suspected fake and counterfeit drugs to regulatory authorities.

\section{References}

1. Wondemagegnehu E. Counterfeit drugs; an overview. A paper presented at the WHO global forum on Pharmaceutical anti counterfeiting. 2002.

2. Counterfeit drugs: Guidelines for the development of measures to combat counterfeit drugs, 1999. WHO/EDM/QSM/99.1.

3. NAFDAC consumer safety bulletin: Special edition, 2004.

4. Spies A R, Dusen V V. Counterfeit drugs; A menace keeps growing. Available at: http://www.Uspharmacist.com . Accessed, May 19, 2006.
5. World Health Organization; Counterfeit medicines: the silent epidemic. Available at: http:// www.who.int Accessed, May 19, 2006.

6. Scientists use scanners to weed out fake drugs. Available at: http://www.postgazette.com/healthscience/20011105fakedrugshe alth3p3.asp. Accessed, May 19, 2006.

7. Rittenburg J. Counterfeit drugs- $A$ clear and present danger. Available at http://www.samedanltd.com/members/archives/pm ps/summer2002.htm Accessed, May 19, 2006.

8. Seiter A. Pharmaceuticals: Counterfeits, Substandard Drugs and Drug Diversion. Health Nutrition and Population Brief No.2. March 2005. Available at www.worldbank.org./healthnutritionand population/HNP briefNo2 Accessed, May 19, 2006.

9. Dondorp AM, Newton PN, Mayxay M, Van Damme W et al. Fake antimalarials in south East Asia are a major impediment to malaria control. Multinational cross- sectional on the prevalence of fake antimalarials .Trop.Med.Int.Health. 2004; 9:12416.

10. Akunyili DN. Strategies employed in combating drug counterfeiting in Nigeria. A paper presented at the WHO Workshop in collaboration with Italian Medicines Agency and The International Federation of Pharmaceutical Manufacturers and Associations, Held in Rome, Italy $15^{\text {th }}-18^{\text {th }}$ Feb. 2006.

11. Polli JE, Hoag SN. Technology vs Fake drugs Us Pharmacist 2004 Feb; 29. Available at www.Uspharmacist.com. Accessed, May 19, 2006.

12. ten Ham M. Health risks of counterfeit pharmaceuticals. Drug Saf. 2003; 26 : 991-7

13. Clark C, Goldberg L. High volume products likely to be the next target for medicine counterfeiters. The Pharmaceutical Journal. 2004; 272:31-32. 